three groups. LVMI (as determined by echocardiography) was greater in hypertensive and borderline hypertensive children than in normotensive children $(P<0.0005$ and $P<0.02$, respectively). Renal volume (as assessed by abdominal ultrasonography) was greater in hypertensive children than in borderline hypertensive or normotensive children $(P<0.002$ and $P<0.0002$, respectively). Both LVMI and log renal volume correlated significantly with systolic blood pressure $(r=0.45$ and $r=0.70$, respectively; $P<0.0001$ for both) and diastolic blood pressure $(r=0.29, P<0.007$ and $r=0.52$, $P<0.0001$, respectively).

LVMI seems to be increased in children with ADPKD and borderline hypertension; therefore, cardiac monitoring and early therapeutic intervention might be advisable for these individuals.

Original article Cadnapaphornchai MA et al. (2008) Increased left ventricular mass in children with autosomal dominant polycystic kidney disease and borderline hypertension. Kidney Int 74: 1192-1196

\section{In-center intermittent peritoneal dialysis is a viable alternative to home treatment}

For patients who cannot tolerate hemodialysis and are unable or unwilling to perform peritoneal dialysis at home, in-center intermittent peritoneal dialysis (IPD) might be the only treatment option available. Although renal registries do not collect data on IPD, outcomes associated with this modality are assumed to be very poor. To address the scarcity of data on this issue, Woywodt et al. conducted a retrospective study of severely ill patients who underwent IPD at a single center in Hanover, Germany.

The study analyzed data from 30 patients who received 7.5-8h daytime sessions of IPD during 1997-2007 (median duration of treatment 7 months, range 1-61 months; mean age at treatment initiation 73.4 years; mean of $>12$ comorbidities per patient). The most common reasons for choosing in-center IPD were congestive heart failure $(n=13)$ and refusal of home therapy $(n=12)$. The leading causes of hospitalization while on IPD were cardiac disease, peritonitis and peripheral vascular disease (in 9, 9 and 8 patients, respectively). The hospitalization rate was low: 1.39 admissions per IPD-year, or a mean of 1.7 admissions per patient. Only 1 episode of peritonitis occurred per 48.8 IPD-months. In total, 14 patients died while on IPD, 9 from sepsis and 5 from cardiac disease.

The authors conclude that rates of peritonitis, hospitalization and survival in IPD are not as poor as is usually assumed and that this modality deserves further attention in renal registries and prospective studies.

Original article Woywodt A et al. (2008) In-center

intermittent peritoneal dialysis: retrospective ten-year singlecenter experience with thirty consecutive patients. Perit Dial Int 28: 518-526

\section{Use of icodextrin for peritoneal dialysis can lead to false diagnosis of hyperglycemia}

The iso-osmotic peritoneal dialysis solution icodextrin (Extraneal ${ }^{\circledR}$, Baxter International, Inc., Deerfield, IL) contains glucose polysaccharides, which are hydrolyzed by a-amylase to maltose, maltotriose and maltotetraose. In patients with diabetes who undergo peritoneal dialysis with this solution, increased concentrations of these metabolites-especially maltose-in the blood can lead to disparities between glucose measurements from finger-stick glucometers and those from laboratory analyses of venous blood.

Ramos et al. compared glucose measurements from four different glucose finger-stick tests with those from a central laboratory, for nine patients with diabetes who required peritoneal dialysis at a single center in Barcelona, Spain. A blood sample from each patient was evaluated by the Ascensia Breeze II (Bayer Corporation, Pittsburgh, PA), the One Touch Ultra ${ }^{\circledR}$ (Johnson \& Johnson, New Brunswick, $\mathrm{NJ}$ ), the Glucocard Memory 2 (Arkray, Inc., Kyoto, Japan) and the Accu-chek Aviva ${ }^{\circledR}$ (Roche Diagnostics, Mannheim, Germany). The first three of these devices measure glucose by a glucose-oxidase-mediated method, while the last one measures glucose by a glucosedehydrogenase-mediated method (this enzyme uses pyrroloquinoline quinone as a cofactor). For all nine patients, measurements from the glucose-oxidase-based devices showed good correlation with laboratory results $(P<0.0002)$. By contrast, the glucosedehydrogenase-based device consistently overestimated glucose levels. 